\title{
Locating-Chromatic Number of Amalgamation of Stars
}

\author{
Asmiati", H. Assiyatun \& E.T. Baskoro \\ Combinatorial Mathematics Research Group, \\ Faculty of Mathematics and Natural Sciences, \\ Institut Teknologi Bandung, Jl. Ganesa 10 Bandung. \\ Email: asmiati308@students.itb.ac.id
}

\begin{abstract}
Let $G$ be a connected graph and $c$ a proper coloring of $G$. For $i=1,2, \ldots, k$ define the color class $C_{i}$ as the set of vertices receiving color $i$. The color code $c_{\Pi}(v)$ of a vertex $v$ in $G$ is the ordered $k$-tuple $\left(d\left(v, C_{1}\right), \ldots, d\left(v, C_{k}\right)\right)$ where $d\left(v, C_{i}\right)$ is the distance of $v$ to $C_{i}$. If all distinct vertices of $G$ have distinct color codes, then $c$ is called a locating-coloring of $G$. The locating-chromatic number of graph $G$, denoted by $\chi_{L}(G)$ is the smallest $k$ such that $G$ has a locating coloring with $k$ colors. In this paper we discuss the locating-chromatic number of amalgamation of stars $S_{k, m} . S_{k, m}$ is obtained from $k$ copies of star $K_{1, m}$ by identifying a leaf from each star. We also determine a sufficient condition for a connected subgraph $H \subseteq S_{k, m}$ satisfying $\chi_{L}(H) \leq \chi_{L}\left(S_{k, m}\right)$.
\end{abstract}

Keywords: amalgamation of stars; color code; locating-chromatic number.

\section{Introduction}

Let $G$ be a finite, simple, and connected graph. Let $c$ be a proper coloring of a connected graph $G$ using the colors $1,2, \ldots, k$ for some positive integer $k$, where $c(u) \neq c(v)$ for adjacent vertices $u$ and $v$ in $G$. Thus, the coloring $c$ can be considered as a partition $\prod$ of $V(G)$ into color classes (independent sets) $C_{1}, C_{2}, \ldots, C_{k}$, where the vertices of $C_{i}$ are colored by $i$ for $1 \leq i \leq k$. The color code $c_{\Pi}(v)$ of a vertex $v$ in $G$ is the ordered $k$-tuple $\left(d\left(v, C_{1}\right), \ldots, d\left(v, C_{k}\right)\right)$ where $d\left(v, C_{i}\right)=\min \left\{d(v, x) \mid x \in C_{i}\right\}$ for $1 \leq i \leq k$. If all distinct vertices of $G$ have distinct color codes, then $c$ is called a locating-coloring of $G$. A minimum locating-coloring uses a minimum number of colors and this number is called the locating-chromatic number of graph $G$, denoted by $\chi_{L}(G)$.

*Permanent address: Mathematics Departement, Faculty of Mathematics and Natural Sciences, Universitas Lampung, Jl. Brojonegoro no.1 Bandar Lampung, Lampung.

Received January $11^{\text {th }}, 2010$, Revised July $14^{\text {th }}, 2010$, Accepted for publication July $28^{\text {th }}, 2010$. 
The following definition of an amalgamation of graphs is taken from [3]. For $i=1,2, \ldots, k$, let $G_{i}$ be a graph with a fixed vertex $v_{o i}$. The amalgamation $\operatorname{Amal}\left(G_{i}, v_{o i}\right)$ is a graph formed by taking all the $G_{i}$ 's and identifying their fixed vertices. In this paper, we consider the amalgamation of stars. More precisely, for $i=1,2, \ldots, k$, let $G_{i}=K_{1, n_{i}}, n_{i} \geq 1$ where $v_{o i}$ be any leaf (a vertex of

degree 1) of $K_{1, n_{i}}$. We denote the amalgamation of $k$ stars $K_{1, n_{i}}$ by

$S_{k,\left(n_{1}, n_{2}, \ldots, n_{k}\right)}, k \geq 2$. We call the identified vertex as the center (denoted by $x$ ), the vertices of distance 1 from the center as the intermediate vertices (denoted by $\left.l_{i} ; i=1,2, \ldots, k\right)$, and the $j$-th leaf of the intermediate vertex $l_{i}$ by $l_{i j}(j=1,2, \ldots, m-1)$. In particular, when $n_{i}=m, m \geq 1$ for all $i$, we denote the amalgamation of $k$ isomorphic stars $K_{1, m}$ by $S_{k, m}$.

The locating-chromatic number was firstly studied by Chartrand et al. [1]. They determined the locating-chromatic numbers of some well-known classes such as paths, cycles, complete multipartit graphs and double stars. Apart of paths and double stars, the locating-chromatic number of other trees are also considered by Chartrand et al. [2]. They constructed a tree of order $n \geq 5$ with the locating- chromatic number $k$, where $k \in\{3,4, \ldots, n-2, n\}$. They also showed that no tree on $n$ vertices with locating-chromatic number $n-1$.

Based on the previous results, locating-chromatic number of amalgamation of stars have not been studied. Motivated by this, in this paper we determine the locating-chromatic number of amalgamation of stars.

Beside that, we also discuss the monotonicity property of the locatingchromatic number for the class of amalgamation of stars. Clearly, the locatingchromatic number of a star $K_{1, n}$ is $n+1$, for any $n$ (since all vertices must have different color codes). Since any connected subgraph $H$ of $K_{1, n}$ is also a star with small size, then we clearly have $\chi_{L}(H) \leq \chi_{L}\left(K_{1, n}\right)$. However in general for any connected subgraph $H \subseteq G$, the locating-chromatic number of $H$ may not be necessarily smaller or equal to the locating-chromatic number of $G$.

In this paper, we also investigate the monotonicity property of the locatingchromatic number for amalgamation of stars, $S_{k, m}$. We derive a sufficient condition for a connected subgraph $H \subseteq S_{k, m}$ satisfying $\chi_{L}(H) \leq \chi_{L}\left(S_{k, m}\right)$. 
The following results were proved by Chartrand et al. in [1]. The set of neighbours of a vertex $v$ in $G$ is denoted by $N(v)$.

Theorem 1.1. Let $c$ be a locating-coloring in a connected graph $G$. If $u$ and $v$ are distinct vertices of $G$ such that $d(u, w)=d(v, w)$ for all $w \in V(G)-\{u, v\}$, then $c(u) \neq c(v)$. In particular, if $u$ and $v$ are non adjacent vertices of $G$ such that $N(u)=N(v)$, then $c(u) \neq c(v)$.

Corollary 1.1. If $G$ is a connected graph containing a vertex adjacent to $k$ leaves of $G$, then $\chi_{L}(G) \geq k+1$.

\section{$2 \quad$ Main Results}

We first prove some lemmas regarding the properties of locating-chromatic number of amalgamation of stars. From now on $S_{k, m}$ denotes the amalgamation of $k$ isomorphic stars $K_{1, m}$.

Lemma 2.1. For $k \geq 2, m \geq 2$, let $c$ be a proper coloring of $S_{k, m}$, using at least $m$ colors. The coloring $c$ is a locating-coloring if and only if $c\left(l_{i}\right)=c\left(l_{n}\right), i \neq n$ implies $\left\{c\left(l_{i j}\right) \mid j=1,2, \ldots, m-1\right\}$ and $\left\{c\left(l_{n j}\right) \mid j=1,2, \ldots, m-1\right\}$ are distinct.

Proof. Let $P=\left\{c\left(l_{i j}\right) \mid j=1,2, \ldots, m-1\right\}$ and $Q=\left\{c\left(l_{n j}\right) \mid j=1,2, \ldots, m-1\right\}$. Let $c$ be a locating-coloring of $S_{k, m}, k \geq 2, m \geq 2$ using at least $m$ colors and $c\left(l_{i}\right)=c\left(l_{n}\right)$, for some $i \neq n$. Suppose that $P=Q$. Because $d\left(l_{i}, u\right)=d\left(l_{n}, u\right)$ for every $u \in V \backslash\left\{\left\{l_{i j} \mid j=1,2, \ldots, m-1\right\} \cup\left\{l_{n j} \mid j=1,2, \ldots, m-1\right\}\right\}$ then the color codes of $l_{i}$ and $l_{n}$ will be the same. So $c$ is not a locating-coloring, a contradiction. Therefore $P \neq Q$.

Let $\Pi$ be a partition of $V(G)$ into color classes with $|\Pi| \geq m$. Consider $c\left(l_{i}\right)=c\left(l_{n}\right), i \neq n$. Since $P \neq Q$, there are color $x$ and color $y$ such that $(x \in P, x \notin Q)$ and $(y \in P, y \notin Q)$. We will show that color codes for every $v \in V\left(S_{k, m}\right)$ is unique.

- Clearly, $c_{\Pi}\left(l_{i}\right) \neq c_{\Pi}\left(l_{n}\right)$ because their color codes differ in the $x$ thordinate and $y$ th-ordinate. 
- If $c\left(l_{i j}\right)=c\left(l_{n s}\right)$, for some $l_{i} \neq l_{n}$, we will show that $c_{\Pi}\left(l_{i j}\right) \neq c_{\Pi}\left(l_{n s}\right)$. We divide into two cases.

Case 1. If $c\left(l_{i}\right)=c\left(l_{n}\right)$ then by the premise of this theorem, $P \neq Q$. So $c_{\Pi}\left(l_{i j}\right) \neq c_{\Pi}\left(l_{n s}\right)$.

Case 2. Let $c\left(l_{i}\right)=r_{1}$ and $c\left(l_{n}\right)=r_{2}$, with $r_{1} \neq r_{2}$. Then $c_{\Pi}\left(l_{i j}\right) \neq c_{\Pi}\left(l_{n s}\right)$ because their color codes are different at least in the $r_{1}$ th-ordinate and $r_{2}$ th-ordinate.

- If $c\left(l_{i}\right)=c\left(l_{n j}\right), l_{i} \neq l_{n}$, then $c_{\Pi}\left(l_{i}\right)$ contains at least two components of value 1 , whereas $c_{\Pi}\left(l_{n j}\right)$ contains exactly one component of value 1. Thus $c_{\Pi}\left(l_{i}\right) \neq c_{\Pi}\left(l_{n j}\right)$.

- If $c(x)=c\left(l_{i j}\right)$, then color code of $c_{\Pi}(x)$ contains at least two components of value 1 , whereas $c_{\Pi}\left(l_{i j}\right)$ contains exactly one component of value 1 . Thus $c_{\Pi}(x) \neq c_{\Pi}\left(l_{i j}\right)$.

From all above cases, we see that the color code for each vertex in $S_{k, m}$ is unique, thus $c$ is a locating-coloring.

Lemma 2.2. Let $c$ be a locating coloring of $S_{k, m}$ using $m+a$ colors and $H(a)=(m+a-1)\left(\begin{array}{c}m+a-1 \\ m-1\end{array}\right), a \geq 0$. Then $k \leq H(a)$.

Proof. Let $c$ be a locating-coloring of $S_{k, m}$ using $m+a$ colors. For fixed $i$, let $c\left(l_{i}\right)$ be a color of intermediate vertex $l_{i}$, then the number color combinations can be used by $\left\{l_{i j} \mid j=1,2, \ldots, m-1\right\}$ is $\left(\begin{array}{c}m+a-1 \\ m-1\end{array}\right)$. Because one color is used for coloring the center $x$, there are $(m+a-1)$ colors for $l_{i}$, for every $i=1,2, \ldots, k$. By Lemma 2.1, the maximum number of $k$ is $(m+a-1)\left(\begin{array}{c}m+a-1 \\ m-1\end{array}\right)=H(a)$. So $k \leq H(a)$. 
The main result of this paper concerns about locating-chromatic number of $S_{k, m}$.

Theorem 2.1. For $a \geq 0, k \geq 2, m \geq 2$, let $H(a)=(m+a-1)\left(\begin{array}{c}m+a-1 \\ m-1\end{array}\right)$. Then,

$$
\chi_{L}\left(S_{k, m}\right)=\left\{\begin{array}{ccc}
m & \text { for } & 2 \leq k \leq H(0), m \geq 3, \\
m+a & \text { for } & H(a-1)<k \leq H(a), a \geq 1 .
\end{array}\right.
$$

Proof. First, we determine the trivial lower bound. By Corollary 1.1, each vertex $l_{i}$ is adjacent to $(m-1)$ leaves, for $i=1,2, \ldots, k$. Thus, $\chi_{L}\left(S_{k, m}\right) \geq m$.

Next, we determine the upper bound of $\chi_{L}\left(S_{k, m}\right)$ for $2 \leq k \leq H(0)=m-1$. Let $c$ be a coloring of $V\left(S_{k, m}\right)$ using $m$ colors. Without loss of generality, we can assign $c(x)=1$ and $c\left(l_{i}\right)=i+1$ for $i=1,2, \ldots, k$. To make sure that the leaves will have distinct color code, we assign $\left\{l_{i j} \mid j=1,2, \ldots, m-1\right\}$ by $\{1,2, \ldots, m\} \backslash\{i+1\}$ for any $i$. Then, by Lemma 2.1 , we have that $c$ is a locating-coloring. Thus $\chi_{L}\left(S_{k, m}\right) \leq m$.

Next, we shall improve the lower bound for the case of $k$ such that $H(a-1)<k \leq H(a), a \geq 1$. Since $k>H(a-1)$ then by Lemma 2.2, $\chi_{L}\left(S_{k, m}\right) \geq m+a$. On the other hand if $k>H(a)$ then by Lemma 2.2, $\chi_{L}\left(S_{k, m}\right) \geq m+a+1$. Thus $\chi_{L}\left(S_{k, m}\right) \geq m+a$ if $H(a-1)<k \leq H(a)$.

Next, we determine the upper bound of $\chi_{L}\left(S_{k, m}\right)$ for $H(a-1)<k \leq H(a)$, $a \geq 1$. Without loss of generality, let $c(x)=1$ and color the intermediate vertices $l_{i}$ by $2,3, \ldots, m+a$ in such a way that the number of the intermediate vertices receiving the same color $t$ does not exceed $\left(\begin{array}{c}m+a-1 \\ m-1\end{array}\right)$, for any $t$. We are able to do so because $H(a-1)<k \leq H(a)$. Therefore, if $c\left(l_{i}\right)=c\left(l_{n}\right)$, $i \neq n$ then we can manage $\left\{c\left(l_{i j}\right) \mid j=1,2, \ldots, m-1\right\} \neq\left\{c\left(l_{n j}\right) \mid j=1,2, \ldots, m-1\right\}$. By Lemma 2.1, $c$ is a locating-coloring on $S_{k, m}$. So $\chi_{L}\left(S_{k, m}\right) \leq m+a$ for $H(a-1)<k \leq H(a)$. 
The following figures show minimum locating-colorings on $S_{4,6}$ and $S_{9,3}$.

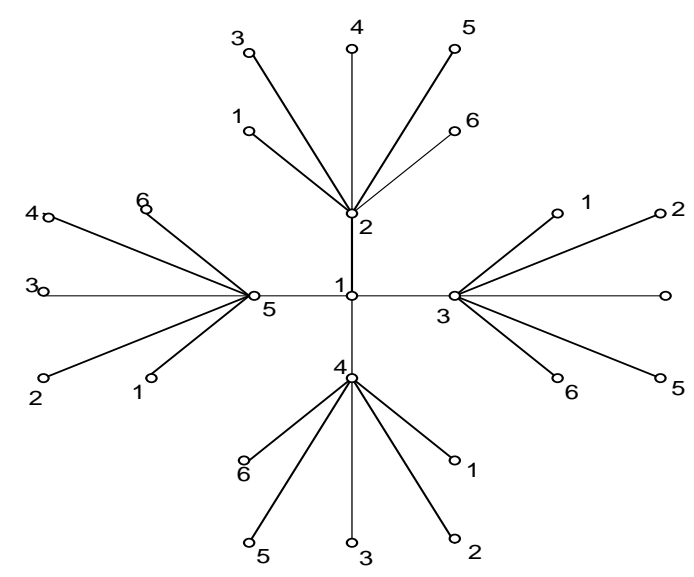

Figure 1 A minimum locating-coloring of $S_{4,6}$.

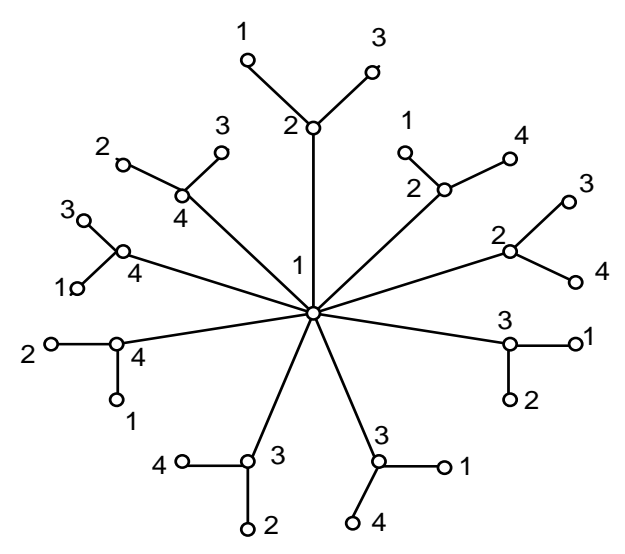

Figure 2 A minimum locating-coloring of $S_{9,3}$.

Next, we discuss the monotonicity property of locating-chromatic number for the amalgamation of stars.

Theorem 2.2 If $2 \leq k \leq m-1$, then $\chi_{L}(G) \leq \chi_{L}\left(S_{k, m}\right)$ for every $G \subseteq S_{k, m}$ and $G \neq K_{1, m}$.

Proof. Let $c$ be a minimum locating-coloring of $S_{k, m}$ obtained from Theorem 2.1. Let $G$ be any connected subgraph of $S_{k, m}$. Define a coloring $c^{\prime}$ on $G$ by 
preserving colors used in $S_{k, m}$ for the corresponding vertices, namely $c^{\prime}\left(v^{\prime}\right)=c(v)$ if $v$ is the corresponding vertex of $v^{\prime}$ in $S_{k, m}$. We show that $c^{\prime}$ is a locating-coloring of $G$.

If there exist $l_{i}, l_{n}$ such that $\left\{c^{\prime}\left(l_{i j}\right) \mid j=1,2, \ldots, r\right\}=\left\{c^{\prime}\left(l_{n j}\right) \mid j=1,2, \ldots, r\right\}$, with $1 \leq r \leq m-1$, then color codes of $l_{i j}$ and $l_{n j}$ for every $j=1,2,3, \ldots, m$ is unique because $c^{\prime}\left(l_{i}\right) \neq c^{\prime}\left(l_{n}\right)$ for every $l_{i} \neq l_{n}$. If $c^{\prime}\left(l_{i}\right)=c^{\prime}\left(l_{n j}\right) \neq c^{\prime}(x)$, then the first component of $c_{\pi}^{\prime}\left(l_{i}\right)$ has value 1 , whereas for $c_{\pi}^{\prime}\left(l_{n j}\right)$ it has value 2 . So color code of $l_{i}$ and $l_{n j}$ are different. Next, if $c^{\prime}(x)=c^{\prime}\left(l_{n j}\right), G \neq P_{3}$ then their color codes are different because $c^{\prime}\left(l_{i}\right) \neq c^{\prime}\left(l_{n}\right)$ for every $l_{i} \neq l_{n}$. For the case $G=P_{3}$, $v_{i} \in V\left(P_{3}\right)$ for each $i$ is colored by 1,2 , and 3 respectively. Because the color codes for every $v \in V(G)$ is unique, then $c^{\prime}$ is a locating-coloring of $G$. So $\chi_{L}(G) \leq \chi_{L}\left(S_{k, m}\right)$ for every $G \subseteq S_{k, m}, G \neq K_{1, m}$.

Let $S_{k,\left(n_{1}, n_{2}, \ldots, n_{k}\right)} \subseteq S_{k, m}$. Define $A=\left\{i \mid n_{i}=1\right\}$. For $k \geq m$, we must restrict subgraphs of $S_{k, m}$ so that satisfy monotonicity property.

Theorem 2.3 If $k \geq m$ and $|A| \leq \chi_{L}\left(S_{k, m}\right)-1$ then $\chi_{L}\left(S_{k,\left(n_{1}, n_{2}, \ldots, n_{k}\right)}\right) \leq \chi_{L}\left(S_{k, m}\right)$.

Proof. Let $k \geq m$ and from Theorem 2.1, we have that $\chi_{L}\left(S_{k, m}\right)=m+a$ for $H(a-1)<K \leq H(a), a \geq 1$. Let $G=S_{k,\left(n_{1}, n_{2}, \ldots, n_{k}\right)}$ be any subgraph obtained from $S_{k, m}$ with $1 \leq n_{i} \leq m$. If $2 \leq n_{i} \leq m$ for each $i$, then color vertices of $G$ follow the proof of Theorem 2.1. Clearly, the coloring of $G$ is a locating-coloring. Otherwise, we have $n_{i}=1$ for some $i$, and so $|A| \geq 1$. If $|A| \leq \chi_{L}\left(S_{k, m}\right)-1$, then the center $x$ is given color $1, l_{i} \in A$ for each $i$ is colored by $2,3, \ldots, \chi_{L}\left(S_{k, m}\right)$, respectively and the colors for the other vertices follow the proof of Theorem 2.1. Observe that the color codes of $l_{i}$ for each $l_{i} \in A$ has value 1 in the 1 thordinate, 0 in the $i$ th-ordinate, and 2 otherwise, these color codes are unique. For the remaining of the vertices, the color codes are also unique as proven in Theorem 2.1. As the result, the coloring of $G$ is a locating-coloring. So $\chi_{L}\left(S_{k,\left(n_{1}, n_{2}, \ldots, n_{k}\right)}\right) \leq \chi_{L}\left(S_{k, m}\right)$. 


\section{References}

[1] Chartrand, G., Erwin, D., Henning, M.A., Slater, P.J. \& Zang. P., The locating-chromatic number of a graph, Bull. Inst. Combin. Appl., 36, pp. 89-101, 2002.

[2] Chartrand, G., Erwin, D., Henning, M.A., Slater \& P.J., Zang. P., Graph of order $n$ with locating-chromatic number $n-1$, Discrete Mathematics, 269, pp. 65-79, 2003.

[3] Carlson, K., Generalized books and $C_{m}$-snakes are prime graphs, Ars Combin., 80, pp. 215-221, 2006. 